\title{
COMMENT ON THE REAL LINE
}

\author{
PAUL R. HALMOS
}

Is it possible to topologize the additive group of the real line so that it becomes a compact topological group? It is the purpose of this note to establish the affirmative answer to this question. While the fact is not deep and the proof not difficult, they are presented here mainly to call attention to the almost completely unsolved problem of algebraic characterization of those abstract abelian groups which can be compact.

(1) If $G$ is any additively written abelian group and $n$ is any positive integer, consider the subgroups $G_{n}^{\prime}$ and $G_{n}^{\prime \prime}$ defined as follows. $G_{n}^{\prime}$ is the set of all $x$ 's in $G$ for which $n x=0 ; G_{n}^{\prime \prime}$ is the set of all $x$ 's which have the form $x=n y$ for some $y$ in $G$. These two subgroups are dual in the sense that if $G$ is a locally compact abelian group and $\Gamma$ is its character group, then the annihilator of $G_{n}^{\prime}$ is $\Gamma_{n}^{\prime \prime}$ and (consequently) the annihilator of $G_{n}^{\prime \prime}$ is $\Gamma_{n}^{\prime}$. (The first statement implies that the annihilator of $\Gamma_{n}^{\prime \prime}$ is $G_{n}^{\prime}$; the second statement follows from this by interchanging the roles of $G$ and $\Gamma$.) To prove this let $\xi=\xi(x)$ be any element of $\Gamma$, and consider the identity $\xi(n x)=n \xi(x)$. Since $\xi(n x)$ vanishes identically if and only if $\xi$ belongs to the annihilator of $G_{n}{ }^{\prime \prime}$, and $n \xi(x)$ vanishes identically if and only if $\xi \in \Gamma_{n}^{\prime}$, the asserted duality follows. It follows also that a locally compact abelian group $G$ is divisible if and only if its character group $\Gamma$ is torsion free, and therefore that $G$ has both properties if and only if $\Gamma$ has.

(2) In a torsion free abelian group the division of an element by an integer is unique (if at all possible). Therefore a torsion free group in which division is always possible admits the additive group of rationals as operators. Consequently such a group is a vector space over the rationals and its algebraic structure is uniquely characterized by its vector dimension, that is by the cardinal number $\alpha$ of elements in a Hamel basis. For the additive group of the real line $\alpha$ is the power of the continuum. The assertion of this note can be proved therefore by exhibiting a compact group $G$ which is both torsion free and divisible and in which there is a Hamel basis having the power of the continuum.

(3) Let $\Gamma$ be the discrete group of rationals and $G$ its compact character group. It follows from (1) that $G$ is divisible and torsion free. The elements $x \in G$ may be thought of as functions $x(\xi)$ from

Received by the editors May 23, 1944. 
$\Gamma$ to the unit interval modulo 1 . If the elements $x_{1}, \cdots, x_{n}$ are rationally dependent then there exist also integers $k_{1}, \cdots, k_{n}$ such that $k_{1} x_{1}(\xi)+\cdots+k_{n} x_{n}(\xi) \equiv 0(\bmod 1)$ for all $\xi \in \Gamma$ and therefore in particular $k_{1} x_{1}(1)+\cdots+k_{n} x_{n}(1) \equiv 0(\bmod 1)$. Since the mapping $x \rightarrow x(1)$ is an onto homomorphism from $G$ to the unit interval modulo 1 , the existence in $G$ of a Hamel basis with power smaller than that of the continuum leads to a contradiction. For then every subset of $G$ with the power of the continuum would contain a rationally dependent finite set and consequently every subset of the unit interval modulo 1 with the power of the continuum would contain a finite set which is integrally dependent modulo 1 . Since this last possibility is absurd, and since the power of $G$ itself is that of the continuum, it follows that a Hamel basis in $G$ has exactly the power of the continuum. The affirmative answer to the question of this note is thus completely established.

SYRACUSE UNIVERSITY 